\title{
Effect of multiple scattering on transition radiation from a dynamic interface
}

\author{
A. F. Barghouty \\ Physics Department, Roanoke College, Salem, Virginia 24153
}

\section{J. Pantazopoulou}

\author{
Physics Department, Virginia Polytechnic Institute and State University, Blacksburg, Virginia 24061
}

(Received 20 August 1990; revised manuscript received 15 May 1991)

\begin{abstract}
The effect of multiple scattering (long-range, small-angle elastic Coulomb collisions) on the radiation field associated with transition radiation from a nonstationary plasmalike medium is addressed with the help of a simple model. The model assumes a relativistic charged particle traversing a single medium that is rapidly and abruptly changing its electromagnetic properties. An averaged diffusion tensor is calculated relativistically to effectively approximate a diminished formation zone in the medium, the effect of which on the differential radiation intensity and angular distribution of transition radiation is studied. It is shown here that multiple scattering is more than a fine-tuning effect, in that it has a marked enhancement effect on the differential yield over a wide and significant frequency range of the emitted photons as well as an appreciable shifting and slowing down of the rapid fall of the maximum intensity angular location over an equally significant angular range. It is suggested that one study the effect in an implosion-explosion process of a laser-prepared medium. Implications to transition radiation from a periodic medium and the more general process of transition scattering in nonlinear media are briefly discussed.
\end{abstract}

\section{INTRODUCTION}

The processes of transition radiation and transition scattering are phenomena of general scope and applications. There are processes of the acoustic as well as the electrodynamic type. Applications have ranged from studies of inhomogeneities in He II to celestial x-ray sources and from transition-radiation based detectors of high-energy charged particles to studies of transition effects in plasmas and other nonlinear media. The processes have received extensive attention by both theorists and experimentalists since 1946 when Ginzberg and Frank [1] first proposed and investigated the mechanism of transition radiation. The interest has been strongly stimulated by challenging and practical applications in addition to the underlying fundamental physics of the phenomenon.

Unlike Čerenkov emission (the optical emission of a charge moving with a constant velocity larger than the phase velocity of light in a medium) transition radiation requires only a change in the parameter $v n / c$, where $n$ is the index of refraction. The simplest process satisfying this condition is when a charge crosses the boundary of vacuum and a medium. As such it is an emission process associated with inhomogeneities (both spatial and temporal) in the medium.

In the case of a relativistic charged particle crossing the interface of two media of differing electromagnetic properties the total radiation measured includes both transition radiation as well as bremsstrahlung. Formal treatments of such problems assume infinite particle trajectories [2-4]. This assumption often leads to a degree of arbitrariness when separating that portion of radiation associated with transition radiation [5-8]. However, effects such as multiple scattering can manifest themselves differently upon the competing mechanisms, thus allowing for separation. It is known [5], for example, that while multiple scattering acts to suppress bremsstrahlung, it actually enhances transition radiation. Earlier treatments $[9,10]$ of this problem, however, concentrated almost exclusively on the case of a static interface between two media. To highlight the effect of multiple scattering it becomes necessary to bring out and treat explicitly the dynamics in the problem.

This can be done by considering a nonstationary plasmalike medium and assuming that over some characteristic time and length the medium changes its electromagnetic properties (dynamic interface) giving rise to transition quanta as relativistic charged particles pass through this interface. In this article we model such a process. In Sec. II we outline the derivation of a simple transition radiation differential intensity formula in this dynamicinterface picture for a plasmalike medium (e.g., electron liquid in metals). In Sec. III we consider the effect of multiple scattering on the differential intensity and derive a modified intensity formula. In Sec. IV we proceed to analyze this effect. We summarize the problem and our results in Sec. V where we propose to study the effect in an implosion-explosion process of a laser-prepared medium and offer some concluding remarks emphasizing implications to transition radiation from a periodic medium and the more general process of transition scattering in nonlinear media.

\section{EMISSION DUE TO DYNAMIC INTERFACE}

In our simple model we allow the plasmalike medium (with characteristic temperature $T$, charged-particle den- 
sity $\rho_{0}$, and dielectric permeability $\epsilon$ ) to expand freely and isotropically with characteristic thermal expansion velocity $\beta_{T}$. We further allow the medium to make a "jump," parametrized by an abrupt change in the value of $\epsilon$. This jump is defined by a "transition zone" with a time scale and size $(\Delta t, \Delta z)$. Due to the jump, a change in the parameter $n \beta$ takes place, $n$ being the index of refraction and $\beta$ the velocity of the traversing charged particle, upon which, a formation zone appears and photons are emitted [2]. (The formation zone is the distance along the particle trajectory after which the separation between the particle and the emitted photon is on the order of the photon wavelength.) This formation zone is characterized by $\left(t_{m}, l_{m}\right)$. Here we assume that spatial and temporal dispersions can be neglected. This is the case whenever the transition zone is much smaller in time and size than the formation zone [2]:

$$
\frac{\Delta t}{t_{m}}, \frac{\Delta t_{d}}{t_{m}}, \frac{\Delta z}{l_{m}}, \frac{\Delta z_{d}}{l_{m}} \ll 1,
$$

where $\left(\Delta t_{d}, \Delta z_{d}\right)$ are the characteristic time and extent over which frequency and spatial dispersions are significant such that the concept of dielectric permeability is not well defined. Equation (2.1) can be cast in one compact form to read

$$
\left.\delta_{\text {c.m. }}\right|_{\substack{\omega=\gamma \omega_{p} \\ \theta=\gamma^{-1}}} \approx \frac{2 \pi\left(\frac{3}{4}\right)^{4} \beta_{T}\left(\Delta t \omega_{p}\right)^{2}}{\gamma^{4}} \ll 1,
$$

where $\omega_{p}$ is the medium plasma frequency $\left(e^{2} \rho_{0} / 4 \pi \epsilon_{0} m\right)^{1 / 2}$. The significance of $\delta_{\mathrm{c} . \mathrm{m}}$. will be shown in what follows. Given Eq. (2.2) we can apply the sharp, static-interface expression for the differential radiation intensity [5]:

$$
\frac{d^{2} I}{d \theta d \omega}=\frac{2 \alpha \hbar}{\pi} \theta^{3}(\omega c)^{2}\left(l_{v}-l_{m}\right)^{2},
$$

where $\omega$ is the emitted photon frequency and $\theta$ is the angle this photon makes with the particle trajectory, to our dynamic-interface picture. Equation (2.3) is for a twomedium process where $l_{v}$ and $l_{m}$ are the coherent lengths in vacuum and medium, respectively, given by [5]

$$
\begin{aligned}
& l_{v} \approx \frac{4 c}{\omega}\left(\gamma^{-2}+\theta^{2}\right)^{-1}, \\
& l_{m} \approx \frac{4 c}{\omega}\left[\gamma^{-2}+\theta^{2}+\frac{\omega_{p}^{2}}{\omega^{2}}\right]^{-1},
\end{aligned}
$$

where $\gamma$ is the Lorentz factor of the traversing particle. In essence, $l_{v}$ and $l_{m}$ determine the size of the formation zone of the radiation field. The formalism is taken in the relativistic limit neglecting multiple scattering the particle may suffer during flight [5].

To check Eq. (2.2), we plot in Fig. 1 contours of the "dispersion parameter" $\delta_{\text {c.m. }}$. as functions of $\gamma$ and time of jump $\Delta t$ for our prepared medium at, e.g., $T=10^{4} \mathrm{~K}$ and $\rho_{0}=10^{18} \mathrm{~m}^{-3}$, calculated in the center-of-mass frame of the changing medium and the traversing particle. Because the radiation intensity drops rapidly beyond a cutoff frequency $[5,11]\left[\gamma \omega_{p}\right.$ in Eq. (2.3)], and peaks

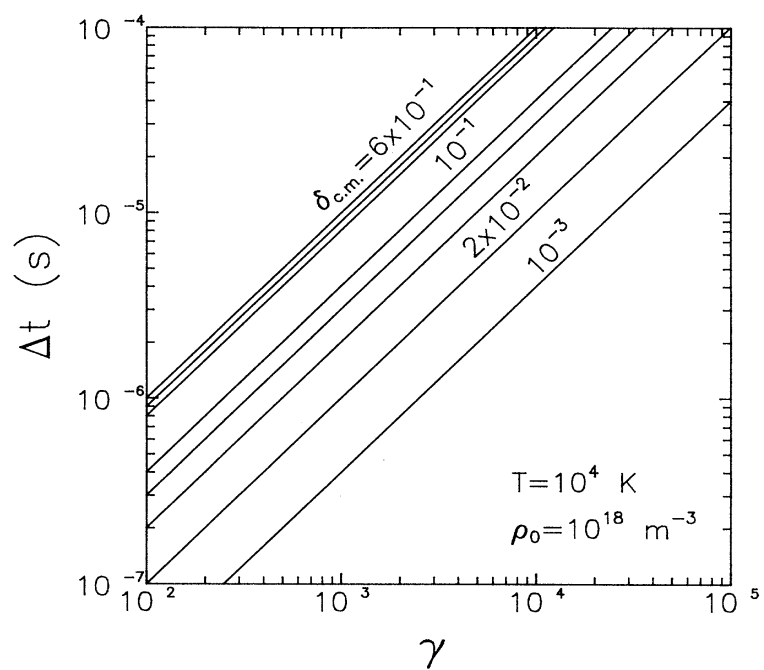

FIG. 1. Contour plot of the "dispersion parameter" $\delta_{\text {c.m. }}$ as functions of $\gamma$ and $\Delta t$ calculated in the center-of-mass frame of the changing medium and the traversing particle, at $\theta=\gamma^{-1}$ and $\omega=\gamma \omega_{p}$.

sharply in the forward region at $\theta \sim \gamma^{-1}$, in Fig. 1 we take $\theta=\gamma^{-1}$ and $\omega=\gamma \omega_{p}$. We treat both $\Delta t$ and $\gamma$ as free parameters in the model. On the basis of Fig. 1 we choose $\Delta t$ to be $\sim 0.5 \mu \mathrm{s}$ and $\gamma=10^{2}$ corresponding to $\delta_{\text {c.m. }}$ on the order of 0.1 , i.e., allowing us to neglect frequency and spatial dispersions.

Although with this choice for $\Delta t$ and $\gamma$ Eq. (2.3) becomes applicable to our model, it is, however, for 1 boundary. In our dynamic-interface picture the presence of a transition zone means 2 boundaries. The first (in time) appears between the expanding medium and the transition zone, the second between the transition zone and vacuum. Hence, at least a three-medium, twoboundary expression is needed to implement a dynamicinterface picture. (The extension from this to a periodic medium, resonance transition radiation, and the more general process of transition scattering will be briefly discussed in Sec. V.) We do this by assuming that no radiation is absorbed and that the particle suffers no deflection during passage through the transition zone (i.e., multiple scattering is taken to be confined to the medium). This allows us to add, taking into account the phase difference, the two (due to two boundaries) radiation fields coherently. And since $I_{\text {tot }} \propto A_{\text {tot }}^{2}$, where $A$ is amplitude, the total differential intensity is now written

$$
d I_{\text {tot }}=\left|d I_{m, T}^{1 / 2}+d I_{T, v}^{1 / 2} e^{-i \varphi}\right|^{2},
$$

where $d I_{m, T}$ denotes the differential intensity due to transition from medium to transition zone given by Eq. (2.3) and $d I_{T, v}$ from transition zone to vacuum. $\varphi$ is the phase difference between the radiation fields emitted at the two boundaries:

$$
\varphi=\frac{2 \Delta z}{l_{T}}
$$


where $l_{T}$ is the coherent length in the transition zone given by

$$
l_{T} \approx \frac{4 c}{\omega}\left[\gamma^{-2}+\theta^{2}+\frac{\bar{\omega}_{p}^{2}}{\omega^{2}}\right]^{-1},
$$

where $\bar{\omega}_{p}$ is the mean medium plasma frequency averaged over the transition zone. Equation (2.6) is a manifestation of the formation zone effect [3]. Thus Eq. (2.3) now becomes

$$
\frac{d^{2} I}{d \theta d \omega}=\frac{2 \alpha \hbar}{\pi} \theta^{3}(\omega c)^{2}\left(l_{m}-l_{T}\right)^{2}\left|1+\frac{l_{T}-l_{v}}{l_{m}-l_{T}} e^{-2 i \Delta z / l_{T}}\right|^{2} .
$$

It is readily seen that Eq. (2.8) reduces to Eq. (2.3) in the limit $\Delta z \rightarrow 0$ (note that $l_{T} \rightarrow l_{v}$ in that limit).

To calculate $l_{T}$, we first calculate the mean chargedparticle density averaged over the size and duration of the transition zone. To that end, we assume our medium has an initial Gaussian distribution for the density [12]

$$
\rho(r ; t=0)=\rho_{0} e^{-r^{2} / r_{0}^{2}},
$$

unfolding as

$$
\rho(r ; t)=\rho_{0} \frac{r_{0}^{3}}{r^{3}(t)} e^{-r^{2} / r^{2}(t)},
$$

where $r(t)=\left(r_{0}^{2}+\bar{\beta}_{T}^{2} t^{2}\right)^{3 / 2}$ and $\bar{\beta}_{T}^{2}=2 / 3 \beta_{T}^{2} . \quad r_{0}$ can be chosen such that the medium is optically thin and in thermal equilibrium with radiation; by requiring that the bremsstrahlung energy loss rate is much smaller than the black-body rate. For our medium this translates to $r_{0} \gg 3 \times 10^{-9} \mathrm{~m}$, which is the limit below which reabsorption of radiation must be taken into account. On the basis of this criterion we take $r_{0} \sim 10^{-4} \mathrm{~m}$ (the third and last free parameter in the model). Averaging over the transition zone then gives [13]

$$
\bar{\rho}=\frac{3}{2} \rho_{0}\left(y^{3}+3 y^{2}+y\right)^{-1}\left(\frac{\sqrt{\pi}}{2} \operatorname{erf}(x)-x e^{-x}\right),
$$

for the mean charged-particle density where $y=\bar{\beta}_{T} \Delta t / r_{0}, x=\bar{\beta}_{T} \Delta t / r(t)$, and erf is an error function. Given Eq. (2.11) we can calculate the medium mean plasma frequency during the transition zone in order to approximate the coherent length $l_{T}$ in that zone, which also appears in the phase difference between the two radiation fields [exponent in the last term of Eq. (2.8)]. Equation (2.8) then gives the differential radiation intensity in the case of a dynamic interface (three-medium, two-boundary process), but includes no multiple scattering.

\section{EFFECT OF MULTIPLE SCATTERING}

Next, we consider the effect of multiple scattering on the radiation field. By multiple scattering, here we mean multiple long-range, small-angle elastic Coulomb collisions between the particles of the medium and the traversing particle. Such collisions will act in such a way to withdraw the traversing particle from the formation zone by diminishing the coherent length $l_{m}$ in the medium $[5,9,10]$. Consequently, the "new" coherent length $l_{m}^{\prime}$ will be smaller on scattering than in its absence, thus enhancing the intensity of radiation [Eq. (2.8)]. In contrast, this diminishing of the coherent length distorts significantly radiation characteristics from bremsstrahlung $[5,10]$. To quantify this statistically we assume uncorrelated (in position) near (within a Debye length) collisions, allowing us (neglecting bremsstrahlung) to write

$$
l_{m}^{\prime} \approx \frac{4 c}{\omega}\left[\gamma^{-2}+\theta^{2}+\left\langle\theta_{s}^{2}\right\rangle+\frac{\omega_{p}^{2}}{\omega^{2}}\right]^{-1},
$$

where $\left\langle\theta_{s}^{2}\right\rangle$ is the mean square of the scattering angle over the length $l_{m}$. From Eqs. (2.8) and (3.1) we see $[5,11]$ that a considerable contribution to the radiation intensity comes from the radiation angles $\theta$ up to $\left(\left\langle\theta_{s}^{2}\right\rangle\right)^{1 / 2}$. We can express the new coherent length $l_{m}^{\prime}$ in terms of the old [13] as

$$
l_{m}^{\prime}=\eta l_{m}=\frac{1}{1+\left\langle\theta_{s}^{2}\right\rangle\left(\gamma^{-2}+\theta^{2}+\omega_{p}^{2} / \omega^{2}\right)^{-1}} l_{m},
$$

with the dimensionless parameter $\eta$ describing the degree of withdrawnness from the formation zone. In the limit $\left\langle\theta_{s}^{2}\right\rangle \rightarrow 0, \eta \rightarrow 1$. In the following we give a prescription to estimate $\eta$.

To calculate $\left\langle\theta_{s}^{2}\right\rangle$ we look at the traversing particle and an average medium particle interacting in their center-of-mass frame (the following prescription takes both particles to be electrons) and treat that relativisitically as an elastic binary collision. Upon each typical collision, the traversing particle is deflected from its original trajectory by the angle $\left(\left\langle\theta_{s}^{2}\right\rangle\right)^{1 / 2}$, which can be written as

$$
\left(\left\langle\theta_{s}^{2}\right\rangle\right)^{1 / 2}=\cos ^{-1}\left[1-\frac{\left\langle\Delta p^{2}\right\rangle}{2 q_{0}}\right),
$$

where $\sqrt{q_{0}}$ is the center-of-mass three-momentum and $\left\langle\Delta p^{2}\right\rangle$ is the mean-square three-momentum transfer. In terms of a characteristic deflection time $t_{D}$ and an average three-velocity change per unit time $\langle\Delta \boldsymbol{\beta}\rangle,\langle\Delta \mathbf{p}\rangle$ can be written as

$$
\langle\Delta \mathbf{p}\rangle=m \gamma_{\mathrm{c} . \mathrm{m} .} t_{D}\langle\Delta \boldsymbol{\beta}\rangle,
$$

with $\gamma_{\text {c.m. }}$ being the center-of-mass Lorentz factor. $\langle\Delta \boldsymbol{\beta}\rangle$ gives a measure of the field strength. In a coordinate system where components parallel to $\beta$ are horizontal components and those perpendicular to $\beta$ are vertical, the diffusion tensor is diagonal [14]:

$$
\left\langle\Delta \beta_{i} \Delta \beta_{j}\right\rangle=\left(\begin{array}{ccc}
\left\langle\Delta \beta_{\|}^{2}\right\rangle & 0 & 0 \\
0 & \frac{1}{2}\left\langle\Delta \beta_{\perp}^{2}\right\rangle & 0 \\
0 & 0 & \frac{1}{2}\left\langle\Delta \beta_{\perp}^{2}\right\rangle
\end{array}\right) .
$$

In terms of the diagonal elements, $\left\langle\Delta p^{2}\right\rangle$ is now written

$$
\left\langle\Delta p^{2}\right\rangle=\left(m \gamma_{\mathrm{c} . \mathrm{m} .} t_{D}\right)^{2}\left(\left\langle\Delta \beta_{\|}^{2}\right\rangle+\left\langle\Delta \beta_{\perp}^{2}\right\rangle\right),
$$

where $t_{D}$ is now given by 


$$
t_{D}=\frac{\beta_{\mathrm{c} . \mathrm{m} .}^{2}}{\left\langle\Delta \beta_{\perp}^{2}\right\rangle} .
$$

The diffusion tensor is now calculated assuming spherically symmetric velocity and space-charge distributions in configuration and momentum space, respectively, e.g., a Maxwellian distribution for the medium particles:

$$
f\left(\beta_{m}\right)=\rho_{0}\left(\frac{m}{2 \pi k T}\right)^{3 / 2} e^{-m \beta_{m}^{2} / 2 k T},
$$

where $k$ is Boltzmann's constant and $\beta_{m}$ is the typical medium-particle velocity; this assumption allows us to integrate over all medium particles. Performing such integration [14] leads to the following expressions for the diagonal terms:

$$
\left\langle\Delta \beta_{\|}^{2}\right\rangle=\frac{A_{D}}{\beta_{\text {c.m. }}} S(\zeta)
$$

and

$$
\left\langle\Delta \beta_{\perp}^{2}\right\rangle=\frac{A_{D}}{\beta_{\text {c.m. }}}[\operatorname{erf}(\zeta)-\mathcal{S}(\zeta)],
$$

where $A_{D}$ is the diffusion constant given by

$$
A_{D}=\frac{e^{4}}{2 \pi \epsilon_{0}^{2} m^{2}} \rho_{0} \ln \Lambda,
$$

and $\Lambda$ is defined by

$$
\Lambda=2 \pi \epsilon_{0} \frac{m \lambda_{D} \beta_{\mathrm{c.m} .}^{2}}{e^{2}},
$$

where $\lambda_{D}$ is the Debye length $\left(4 \pi \epsilon_{0} k T / e^{2} \rho_{0}\right)^{1 / 2}$, and $\beta_{\text {c.m. }}$ is the center-of-mass velocity. Here we take the typical collision with an impact parameter equal to $\lambda_{D}$. The function $\mathcal{G}$ in Eqs. (3.9) and (3.10) is defined in terms of the error function as

$$
\mathcal{S}(\zeta)=\frac{\operatorname{erf}(\zeta)-\xi \frac{d \operatorname{erf}(\zeta)}{d \zeta}}{2 \xi^{2}} .
$$

The argument of both $\mathcal{G}$ and the error function $\xi=\sqrt{m / 2 k T} \beta_{\text {c.m. }}$ is, of course, the same dimensionless quantity appearing in the Maxwellian distribution since it is the symmetry and kernel of the distribution that allowed us to calculate (on the average) the diffusion tensor in a simple and straightforward fashion [14].

Equations (3.3)-(3.13) offer a simple prescription to estimate the root mean square of the scattering angle in the center-of-mass frame using an averaged diffusion tensor to estimate the dynamical friction the traversing particle experiences due to its interactions with the medium particles. Once this angle is transformed to the laboratory frame it gives a measure [Eq. (3.2)] of the degree of withdrawnness from the formation zone. Equation (2.8) is now written

$$
\frac{d^{2} I}{d \theta d \omega}=\frac{2 \alpha \hbar}{\pi} \theta^{3}(\omega c)^{2}\left(l_{m}^{\prime}-l_{T}\right)^{2}\left|1+\frac{l_{T}-l_{v}}{l_{m}^{\prime}-l_{T}} e^{-2 i \Delta z / l_{T}}\right|^{2},
$$

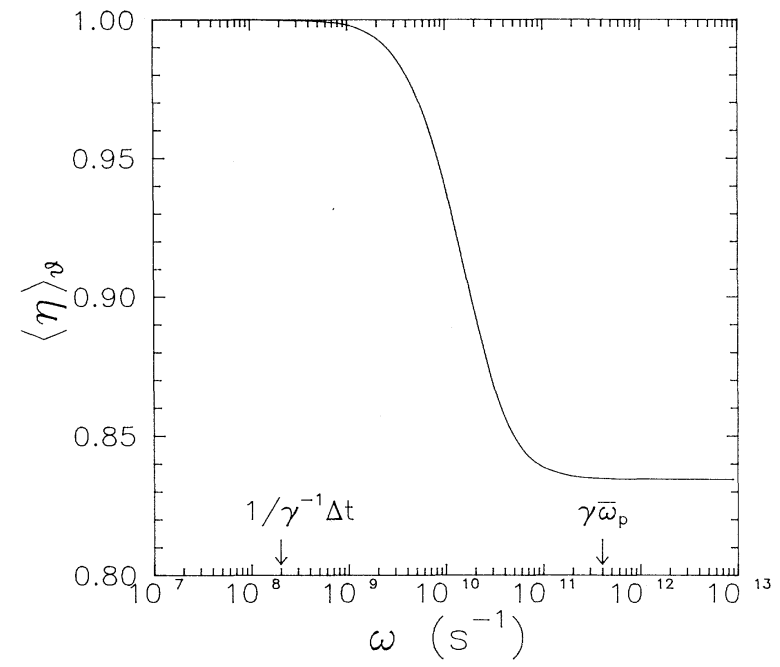

FIG. 2. Angle-integrated $\langle\eta\rangle_{\theta}$ (parametrizing the degree of withdrawnness from the formation zone) as a function of the frequency of the emitted photon.

with $l_{m}^{\prime}$ defined by Eq. (3.2). Hence Eq. (3.14) describes the radiation intensity in the case of a dynamic-interface including a diminished formation zone due to multiple scattering. Next we apply the above prescription to study the effect of a diminished formation zone on the differential radiation intensity and angular distribution of transition radiation from a dynamic interface.

\section{RESULTS}

Before analyzing the effect on the intensity and angular distribution it helps to first isolate the parameter $\eta$ that parametrizes the diminished formation zone and look at its dependence on the angle and frequency of the emitted photon in the laboratory frame. Plotted in Fig. 2 is the

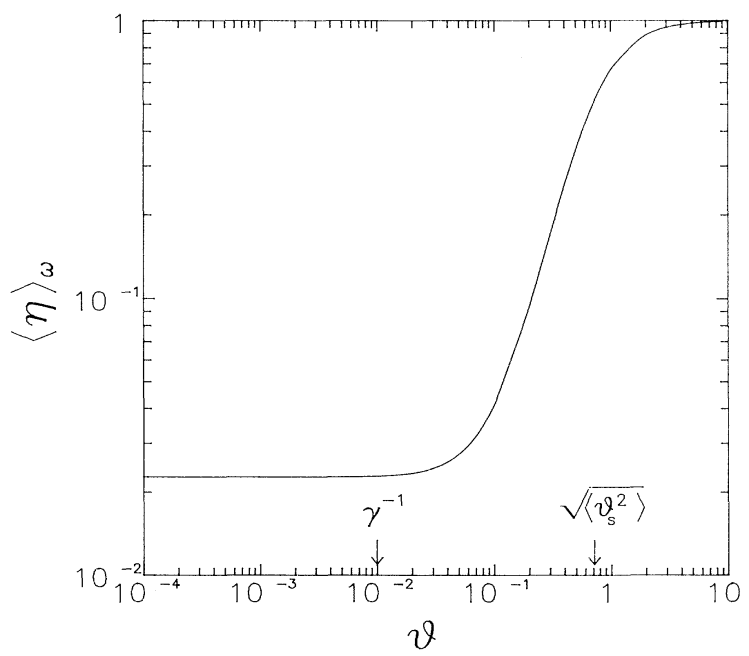

FIG. 3. Frequency-integrated $\langle\eta\rangle_{\omega}$ as a function of the angle of the emitted photon. 
angle-integrated $\langle\eta\rangle_{\theta}\left[=\int_{0}^{2 \pi} d \theta \eta(\omega, \theta) / 2 \pi\right]$ versus the frequency of the emitted photon. The integration is done numerically using Gaussian quadrature. We see that $\eta$ begins to deviate from unity (paremetrizing a nondiminished formation zone) for frequencies above a threshold frequency $1 / \gamma^{-1} \Delta t$. The effect is a smooth and parametric function of frequency over the wide range $\left(1 / \gamma^{-1} \Delta t, \gamma \bar{\omega}_{p}\right)$ It appears to saturate and persist for frequencies above $\gamma \bar{\omega}_{p}$, not inconsistent with earlier qualitative assessments on the effect of multiple scattering [9-11]. As for $\eta$ 's angular dependence, in Fig. 3 we plot the frequency-integrated $\langle\eta\rangle_{\omega}\left[=\int_{0}^{\gamma \omega_{p}} d \omega \eta(\omega, \theta) / \gamma \omega_{p}\right]$ versus the photon angle. For angles below and up to $\gamma^{-1}, \eta$ is far from unity indicating strong diminishing effect on the formation zone. Over the range $\left(\gamma^{-1},\left(\left\langle\theta_{s}^{2}\right\rangle\right)^{1 / 2}\right)$ the same smooth parametric behavior is seen as in the frequency dependence. Thus Figs. 2 and 3 suggest a diminishing effect on the formation zone due to multiple scattering over the frequency range $(1 /$ $\left.\gamma^{-1} \Delta t, \gamma \bar{\omega}_{p}\right)$ and the angular range $\left(\gamma^{-1},\left(\left\langle\theta_{s}^{2}\right\rangle\right)^{1 / 2}\right)$ should be evident, i.e., forward emission for frequencies above $\gamma \bar{\omega}_{p}$.

With this in mind, we plot in Fig. 4 the differential intensity versus frequency. The dashed curve is calculated for the case $\eta=1$, while the solid curve is with $\eta$ as defined by Eq. (3.2) and depicted by Fig. 2. As Fig. 2 suggested earlier, we see here a diminished formation zone beginning to affect the intensity for frequencies at and above $\gamma\left(1 / \gamma^{-1} \Delta t\right)=\gamma^{2} / \Delta t$. At and beyond the cutoff frequency of $\gamma \bar{\omega}_{p}$, the dashed curve begins to follow a logarithmic-law dependence, whereas the solid curve appears to drop much slower and saturate. (The saturation much beyond $\gamma \bar{\omega}_{p}$ here is an artifact resulting from the energy consideration in $\boldsymbol{\eta}$. Once a properly normalized $\boldsymbol{\eta}$ is derived, the solid curve is expected to drop very rapidly at frequencies still, however, above $\gamma \bar{\omega}_{p}$.) With regard

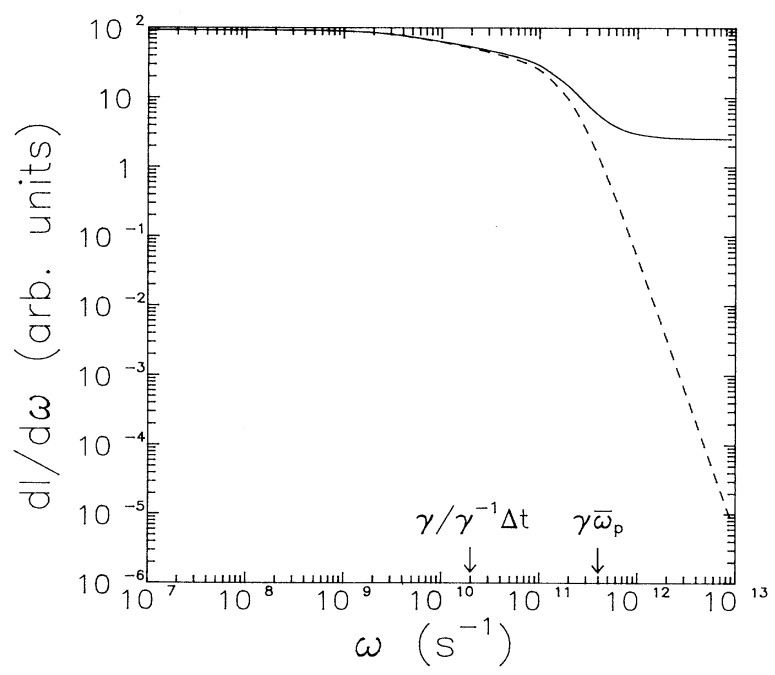

FIG. 4. Differential radiation intensity, Eq. (3.14), vs frequency of the emitted photon. Dashed curve is for the case $\eta=1$ and solid curve is for $\eta$ calculated using Eq. (3.2).

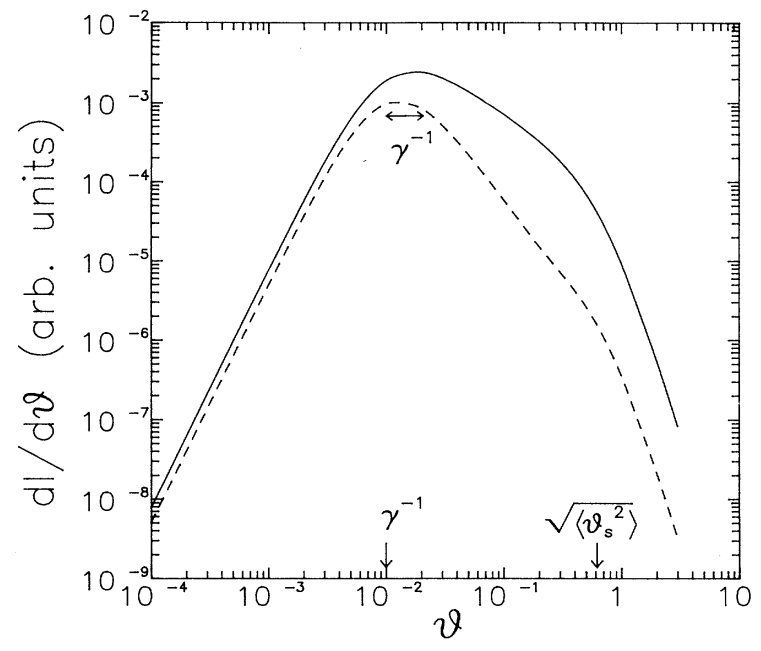

FIG. 5. Differential radiation intensity vs angle of the emitted photon. Dashed and solid curves are as described in Fig. 4 and Sec. IV.

to the angular distribution, plotted in Fig. 5 is the differential yield versus angle of the emitted photon. The dashed curve again is calculated for the case $\eta=1$ and the solid curve is with $\eta$ as defined by Eq. (3.2). Similarly, we see here what Fig. 3 earlier suggested for the range $\left(\gamma^{-1},\left(\left\langle\theta_{s}^{2}\right\rangle\right)^{1 / 2}\right)$ over which the effect of a diminished formation zone is evident. Over this range, we see that a diminished formation zone manifests itself in a slower fall of the maximum intensity as $\theta$ grows in addition to the shifting of the location of this maximum towards the right on the order of $\gamma^{-1}$. [The enhancement of the yield for angles much above $\left(\left\langle\theta_{s}^{2}\right\rangle\right)^{1 / 2}$ is again due to the normalization of $\eta$.]

\section{CONCLUDING REMARKS}

To summarize, a simple model has been presented to model emission of photons due to transition radiation from a dynamic interface. In this model, the plasmalike medium is allowed to expand and make a jump in its electromagnetic properties over a characteristic time and length; transition zone. We approximate and parametrize a diminished formation zone due to multiple scattering the traversing particle experiences over the coherent length in the medium. Treating the collisions as relativistic elastic binary (electron-electron in this model) collisions, an averaged diffusion tensor is calculated to estimate the mean scattering angle. This angle is in turn used in the parametrization of the diminished formation zone. We apply this model to study the effect of this diminishing on the intensity and angular distribution of transition radiation from a dynamic interface.

We find that multiple scattering tends to enhance the differential intensity over the frequency range $\left(1 / \gamma^{-1} \Delta t, \gamma \bar{\omega}_{p}\right)$ and extending beyond the cutoff frequency $\gamma \bar{\omega}_{p}$. Also, we find multiple scattering to tend to slow down the rapid fall of the maximum intensity at $\theta \sim \gamma^{-1}$ 
shifting the location of the maximum on the order of $\gamma^{-1}$ and enhancing the intensity over the angular range $\left(\gamma^{-1},\left(\left\langle\theta_{s}^{2}\right\rangle\right)^{1 / 2}\right)$. In that, the effect of multiple scattering on transition radiation studied here in the context of a dynamic-interface model is shown to be more than a fine-tuning effect. It has been shown to have a marked enhancement effect on the differential yield over a wide and significant frequency range of the emitted photons as well as an appreciable shifting and slowing down of the rapid fall of the maximum intensity angular location over an equally significant angular range (forward emission for frequencies above $\gamma \bar{\omega}_{p}$ ).

We propose that the effect can be studied in an implosion-explosion process of a laser-prepared medium [15]. Temperatures on the order of $10^{4} \mathrm{~K}$ and densities on the order of $10^{20} \mathrm{~m}^{-3}$ are not atypical in such processes. More importantly, time scales on the order of $p s$ have been achieved [15]. Under such conditions, an electron beam with the right energy can be used to probe the sudden change in the electromagnetic properties of the exploding medium. Study of the photon emission and angular distribution associated with transition radiation (with its characteristic cutoff frequency and angular peak) should elucidate the signatures of the effect (forward emission above the cutoff frequency).

We believe that this effect not only is of significance for the simpler processes of medium-vacuum or mediummedium transitions, but also to the more involved processes of transition radiation from a periodic medium (resonance transition radiation) and transition scattering and transition effects in nonlinear media. To give but an example of such implications on the latter cases, consider first transition radiation in the presence of periodicity (which is the next step from our dynamic-interface picture). This periodicity leads to resonance effects as fields are coherently added at the various interfaces. Multiple scattering in this case is sure to play an important part since its contribution to the intensity (forward region), for example, clearly differentiates it from the nonlocalized emission associated with permeability waves in a periodic medium. With regard to the more general process of transition scattering in a plasma, for example, interference between transition scattering and Thomson scattering is of importance. And since transition radiation in a periodic medium may be regarded as transition scattering [2], multiple scattering in this case may play a role since the total scattering cross section includes both Thomson and transition scattering as well as their interference, which can be affected by multiple scattering. And since the nonlinear Landau damping depends on this total cross section [16], the role of multiple scattering may not be of insignificance to the study of transition effects in nonlinear media.

\section{ACKNOWLEDGMENTS}

The authors wish to thank Dr. J. Steehler, and Dr. F. Munley, and Mr. A. McKnight of Roanoke College for their discussion and comments during the preparation of the manuscript. The authors acknowledge the helpful insights they have gained regarding laser-fusion and laserprepared media from discussions with Dr. M. H. Nayfeh and Dr. J. Mazumder of the University of Illinois at Urbana-Champaign.
[1] V. L. Ginzburg and I. M. Frank, Zh. Eksp. Teor. Fiz. 16, 15 (1946).

[2] V. L. Ginzburg and V. N. Tsyovich, Phys. Rep. 49, 1 (1979), and references therein.

[3] G. M. Garibyan, Zh. Eksp. Teor. Fiz. 37, 527 (1959) [Sov. Phys. -JETP 10, 372 (1960)].

[4] I. M. Frank, Usp. Fiz. Nauk 87, 189 (1965) [Sov. Phys.Usp. 8, 729 (1966)].

[5] M. L. Ter-Mikaelian, High-Energy Electrodynamic Processes in Condensed Media (Wiley-Interscience, New York, 1972), Chap. 4, and references therein.

[6] G. B. Yodh, X. Artru, and R. Ramaty, Astrophys. J. 181, 725 (1973).

[7] L. Durand, Astrophys. J. 182, 417 (1973).

[8] M. L. Cherry, G. Hartmann, D. Müller, and T. A Prince,
Phys. Rev. D 10, 3594 (1974).

[9] F. G. Bass and V. M. Yakovenko, Usp. Fiz. Nauk 86, 189 (1965) [Sov. Phys. - Usp. 8, 420 (1965)].

[10] G. M. Garibyan, Zh. Eksp. Teor. Fiz. 39, 332 (1960) [Sov. Phys. - JETP 12, 237 (1961)].

[11] G. M. Garibyan and I. Ya. Pomeranchuk, Zh. Eksp. Teor. Fiz. 37, 1828 (1959) [Sov. Phys._JETP 10, 1290 (1960)].

[12] A. Z. Mekjian, Phys. Rev. C 17, 1051 (1978).

[13] A. F. Barghouty, Bull. Am. Phys. Soc. 32, 1552 (1987).

[14] G. Schmidt, Physics of High Temperature Plasmas (Academic, New York, 1979), Chap. 11.

[15] M. H. Nayfeh and J. Mazumder (private communications).

[16] D.G. Swanson, Plasma Waves (Academic, New York, 1989), Chap 8. 